\title{
The Frequency Characteristics of Mixed Power Networks with Partial Undergrounding
}

\author{
Mohamed M. Saied \\ IEEE Senior Member, Professor (Emeritus), Independent Researcher, Giza, Cairo, Egypt \\ Email: m.saied@ieee.org
}

\begin{abstract}
This paper deals with the frequency characteristics of partially undergrounded mixed power networks, which include several cable sections replacing parts of the overhead lines. The analysis yields the voltage and current distributions along the normal and the partially undergrounded lines, as well as the frequency dependence of the networks' input impedance. Moreover, the results will show eventually existing localized voltage and current concentrations. The study suggests an exact direct analytical distributed parameter method for modeling the lines and cable sections. Instead of representing a partially undergrounded line by the cascade connection of two-port networks, this paper proposes the use of a single equivalent nonuniform line. The nonuniform circuit parameters are represented by means of step functions in terms of the co-ordinates along the lines. The resulting expressions are substituted in the simultaneous voltage and current differential equations, for which analytical solutions are available in terms of Mathematica Parametric Functions.
\end{abstract}

Keywords: Power networks, mixed, nonuniform, overhead-cable lines, partial undergrounding, frequency characteristics, resonance, distributions, input impedance

\section{Introduction}

There is a growing interest in the new concept of the partial undergrounding of high voltage networks comprising overhead transmission lines [1-13]. It is defined as the replacement of parts of these lines by adequate underground cable sections. This can lead to considerable advantages such as:

a) The reduced exposure to the adverse environmental and weather conditions

b) The narrower required corridors and right-of-ways

c) The weaker surrounding magnetic field

d) The reduced nontechnical losses, [1-5]

On the other hand, the following disadvantages should be taken into consideration:

a) The resulting increase in the line cost

b) The higher cable charging capacitive reactive power, [1]

c) The cost of eventually required shunt reactors

d) The increased secondary arc currents associated with the single-pole auto-reclosure and singlephase-to-ground faults, [6]

e) The impact of the eventually needed shunt reactive power compensation on the power system's transient stability, [7].

In terms of the frequency analysis, special attention was devoted to the possible effect of the undergrounding on the networks' resonance frequencies which can coincide with those of some switching events [8].

The effect of the cable sections on the electromagnetic transients in high voltage networks has been thoroughly investigated in several references such as [8-13]. In [11], the transient voltage stresses in a $400-\mathrm{kV}$ cable network are analyzed. Results of experimental investigations addressing three types of switching surges (energization, deenergization and a combination of both) are presented. One of the main observations is the possibility of critical low frequency overvoltages resulting from some switching scenarios. This phenomenon is attributed to the inrush current of the compensating shunt reactor. Reference [12] gives a method for studying the electromagnetic transients in power networks as affected 
by the cable sections taking into account the lengths of the cable sections and the connections of the neutral points. It is shown that the ratio of the surge impedances has a significant impact on the voltage and current transients. Reference [13] deals with the analysis of the electromagnetic transients in typical mixed power networks and assesses the effectiveness of using cable sections in reducing the transients in the network components such as transformer substations. It is based on the distributed parameter component modeling in the $s$-domain. The results of a case study involving multi-pulse lightning surges are also presented.

Reference [9] addresses the possible reduction in the networks' resonance frequencies due to the presence of cable sections. This reduction can lead to the serious damage of some power system components. The paper presents the frequency scans of an assumed frequency dependent power network model. The results of two case studies of a 10-Bus power system as well as of an extra high voltage network in Switzerland are discussed. The paper further emphasizes the need for investigating the frequency response of the mixed networks.

This paper is a further step in this direction and has the following main objectives:

a) To suggest an exact and direct analytical solution for the voltage and current distributions along the various lines and cables within mixed power networks

b) To present a method for identifying the frequency dependence of the networks' input impedance

c) To conduct a parameter study in order to demonstrate the effect of the loading conditions and the circuit parameters of the overhead lines and cable sections on the networks' frequency characteristics

d) To investigate the sensitivity of the frequency characteristics and the resonance frequencies to changes in the locations and lengths of the inserted cable sections as well as their circuit parameters.

\section{$2 \quad$ Method of Analysis}

Consider the single line diagram depicted in Fig.1. It shows a sample 400-kV mixed power network comprising two partially undergrounded overhead transmission lines connecting the source at Bus $s$ with the load at Bus 3. Their total lengths are denoted as $L_{1}$ and $L_{2}$. The lengths of the two cable sections 1 and 2 are $w_{1} L_{1}$ and $w_{2} L_{2}$, respectively. The two factors $w_{1}$ and $w_{2}$ express the per unit degree of the line undergrounding and can assume values ranging between zero and one. The value zero represents the case of no cable sections, and the value one describes the theoretical case of full line undergrounding. The midpoints of the two cable sections are located at distances cen $L_{1}$ and $c e n_{2} L_{2}$ measured from the source bus. By changing the four parameters $w_{1}, c e n_{1}, w_{2}, c e n_{2}$ any cable lengths and locations can be modeled.

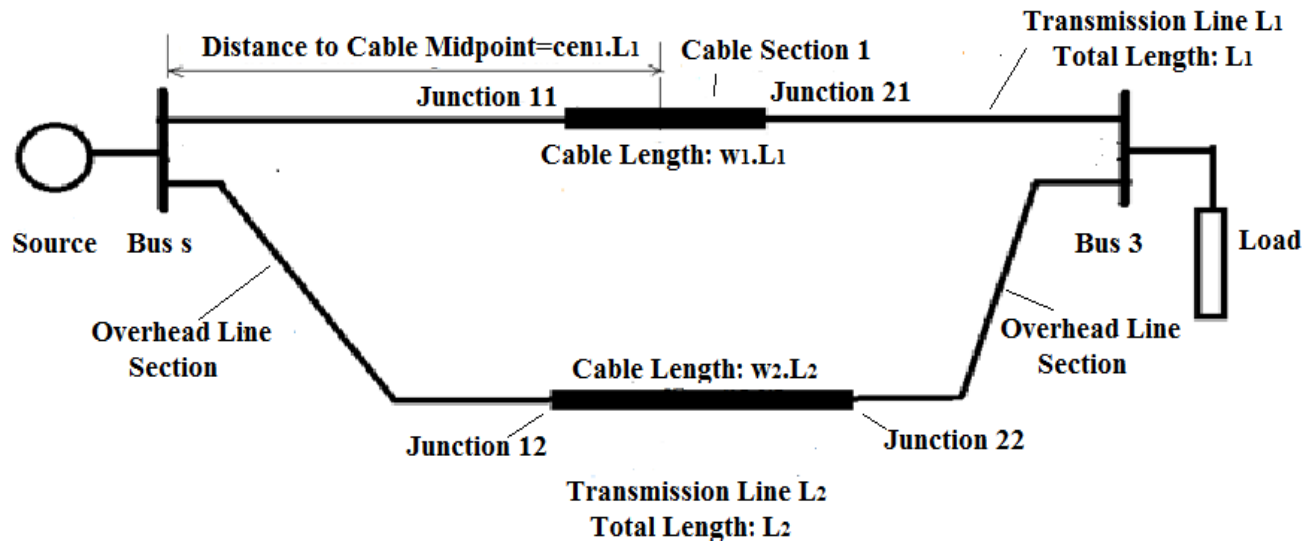

Figure 1. A sample 400-kV partially undergrounded mixed power network.

The electrical parameters of the overhead lines and cable sections of the considered $400-\mathrm{kV}$ network are given in Table I, based on the references $[10,11]$. The shunt conductances are negligibly small. Both lines are assumed $200 \mathrm{~km}$ long. 
Table 1. Data of the 400-kV Overhead Lines and Underground Cables Adopted from the References [10, 11].

\begin{tabular}{lcc}
\hline Parameter & Overhead Line & Underground Cable \\
\hline Resistance Ohm $/ \mathrm{km}$ & 0.026 & 0.020 \\
Inductive Reactance Ohm $/ \mathrm{km}(50-\mathrm{Hz})$ & 0.316 & 0.231 \\
Shunt Capacitance microF $/ \mathrm{km}$ & 0.01137 & 0.1786 \\
\hline
\end{tabular}

The classical way of analyzing this sample network is to represent each of the overhead or cable sections by a two-port network, as shown in Fig.2.

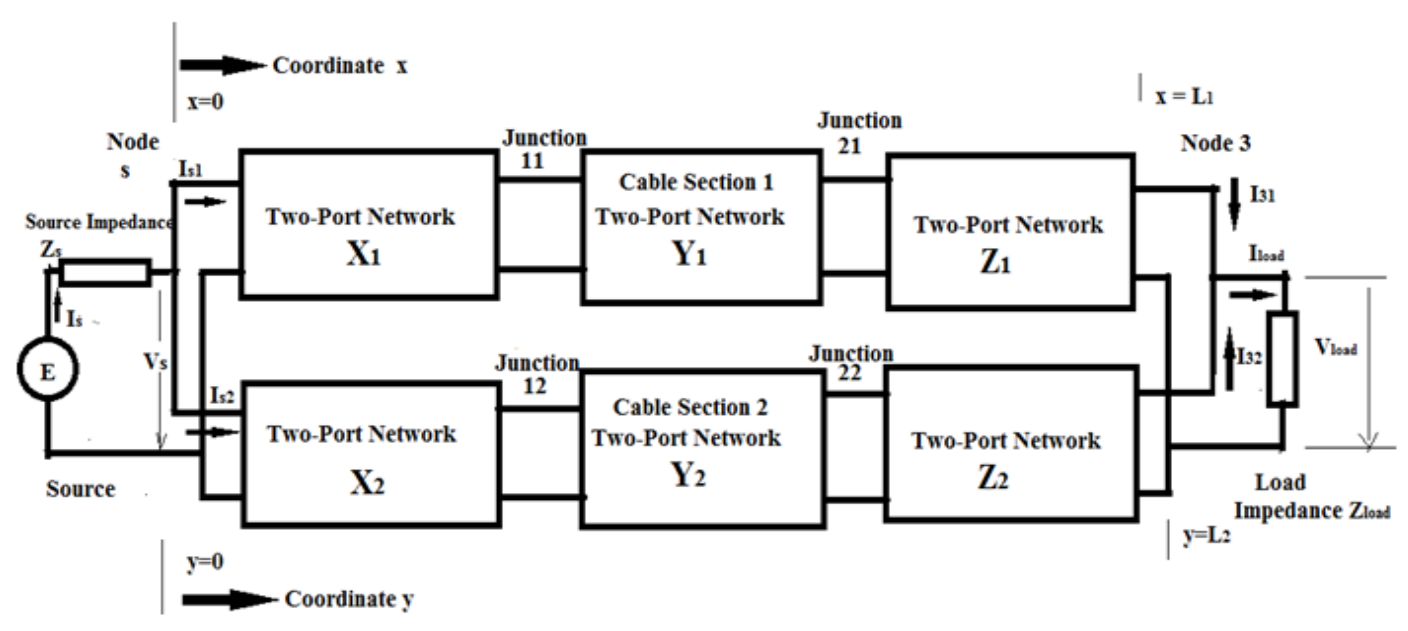

Figure 2. The two-port representation of the sample network.

The upper partially undergrounded line has the two overhead line sections $X_{1}$ and $Z_{1}$ while the twoport network $Y_{1}$ describes the cable section. Similar notations are used for describing the second line $L_{2}$. The values of the voltages and currents at the source bus $s$ and the load bus 3 and at the four junction points denoted as J11, J21, J12 and J22 in Fig.2 can be derived using the algebraic manipulations of the $24(A B C D)$ constants [14]. For any given frequency, this will require the solution of 16 simultaneous complex algebraic equations. From the conditions at the junction points, the internal voltage and current distributions at any point within each of the 6 sections can then be obtained. It should be noted that each of the six two-ports represents a uniform line with constant values of the electrical circuit parameters per unit length.

This paper suggests a more efficient alternative approach to the analysis of partially undergrounded networks. It is based on representing each of the lines $L_{1}$ and $L_{2}$ by a single nonuniform line, i.e. a line with location dependent circuit parameters. The plots in Fig.3 illustrate the case of a $200 \mathrm{~km} 400-$ $\mathrm{kV}$ partially undergrounded line $L_{1}$ with a $40-\mathrm{km}$ cable section (i.e. $w_{1}=0.2$ ). The cable's midpoint is assumed at the line's center (i.e. $\left.c e n_{1}=0.5\right)$. The plots show the line's resistance $r(x)$, inductance $l(x)$ and capacitance $c(x)$ per meter, as well as the location dependent magnitude of the surge impedance $Z_{o}(x)$, as functions of the coordinate $x$ measured from the source terminal. The step-function $u(x)$ can be utilized in order to derive corresponding closed-form analytical expressions for these parameters. For instance, the expressions for the parameters of this partially undergrounded 200-km line $L_{1}$ are

$$
\begin{aligned}
& r(x)=0.000026[u(x)-u(x-80000)]+0.000020[u(x-80000)-u(x-120000)]+ \\
& 0.000026[u(x-120000)-u(x-200000)]
\end{aligned}
$$




$$
\begin{aligned}
& c(x)=10^{-11}[u(x)-u(x-80000)]+18 \times 10^{-11}[u(x-80000)-u(x-120000)]+ \\
& 10^{-11}[u(x-120000)-u(x-200000)] \\
& l(x)=1.1 \times 10^{-6}[u(x)-u(x-80000)]+7.4 \times 10^{-7}[u(x-80000)-u(x-120000)]+ \\
& 1.1 \times 10^{-6}[u(x-120000)-u(x-200000)]
\end{aligned}
$$

Their units are $\Omega / m, F / m$ and $H / m$, respectively.

The shunt conductance $g(x)$ is assumed zero, and the unit of the co-ordinate $x$ is meter.

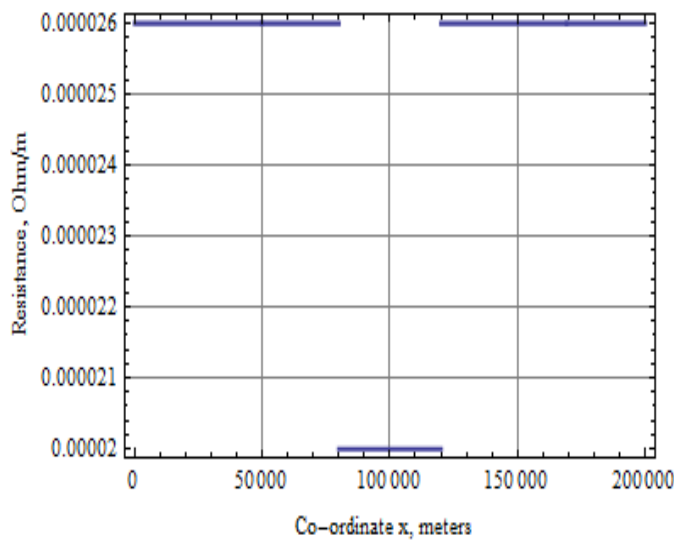

Resistance/m $r(x)$

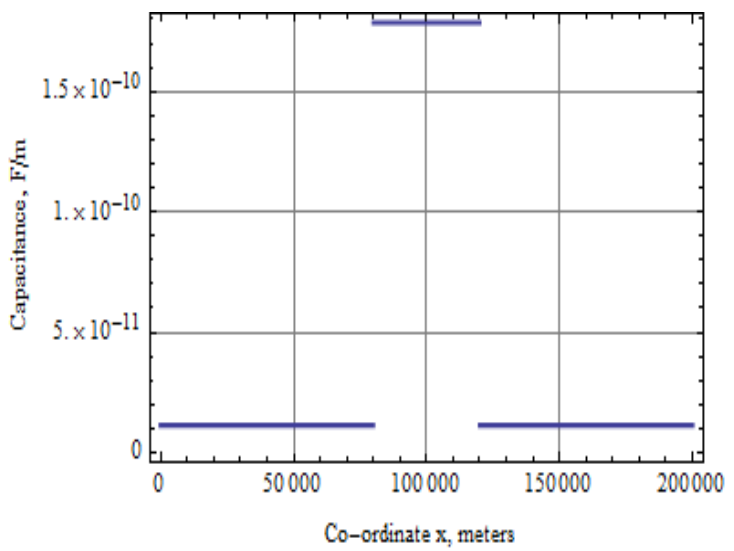

Capacitance/m $c(x)$

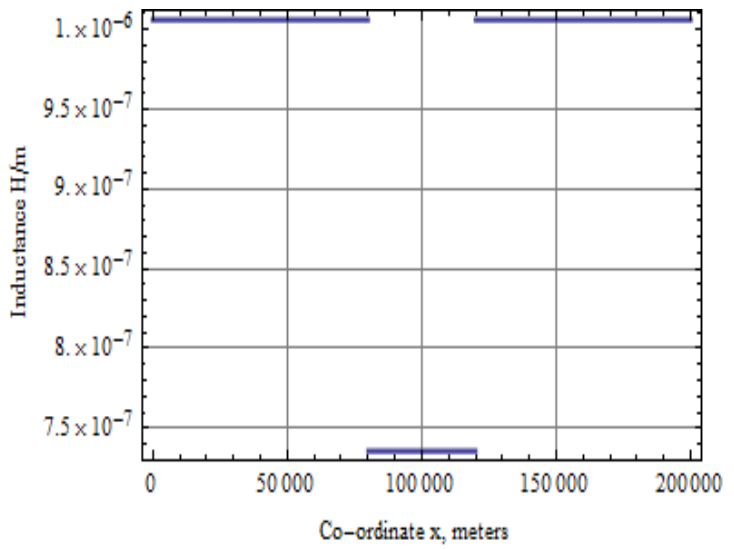

Inductance $/ \mathrm{m} \quad l(x)$

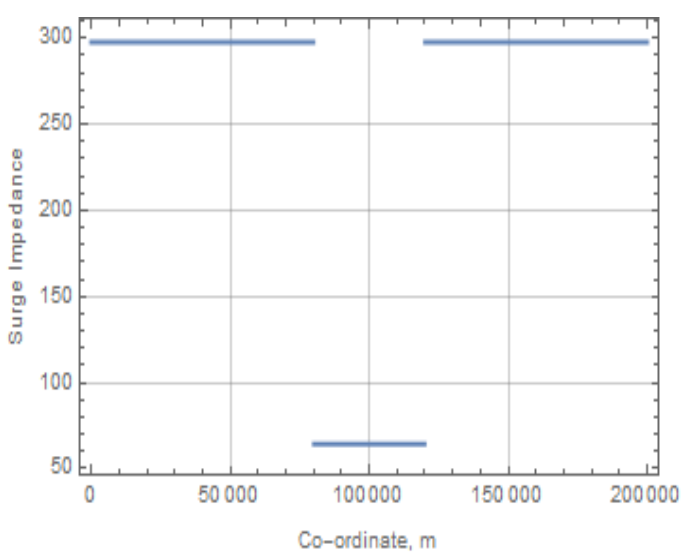

The surge impedance $Z_{o}(x)$

Figure 3. Plots of the circuit parameters per meter: the resistance $r(x)$, the inductance $l(x)$, the capacitance $c(x)$, and the location dependent surge impedance $Z_{o}(x)$ of the equivalent nonuniform line representing $L_{1}$.

Similar plots and equations can be derived for the second (lower) line $L_{2}$.

Each of the two lines depicted in Fig.3 can be described by the following two differential equations. Their independent variable is the coordinate $x$. The Laplace operator $s$, which means the differentiation in the time domain as well as the complex frequency, is treated here as a parameter.

$$
\frac{d v_{1}(x)}{d x}=-i_{1}(x) \cdot r_{1}(x)-i_{1}(x) \cdot s \cdot l_{1}(x)=-i_{1}(x) \cdot\left[r_{1}(x)+s \cdot l_{1}(x)\right]
$$

and

$$
\frac{d i_{1}(x)}{d x}=-v_{1}(x) \cdot g_{1}(x)-v_{1}(x) \cdot s \cdot c_{1}(x)=-v_{1}(x) \cdot\left[g_{1}(x)+s \cdot c_{1}(x)\right]
$$


Two other similar equations can be written for the second line $L_{2}$. The associated boundary conditions can be obtained by applying the Kirchhoff current and voltage laws at each line's source and load terminals. In the sinusoidal domain, $s=j \omega$.

A direct solution technique is available using the Mathematica software (NDSolve). The proposed direct method requires much less computation burden compared with the classical numerical solutions.

The four differential equations, two for each line, could be directly solved by using the Mathematica command (ParametricNDSolve). The output will give the solution in the form of four Parametric Functions for the two voltages and two currents at any point in terms of its co-ordinate and the complex frequency $s$. More details are available in $[15,16]$.

\section{Sample Results}

The following results refer to the network shown in Fig.1 including the two partially undergrounded lines $L_{1}$ and $L_{2}, 200-\mathrm{km}$ long each. $L_{1}$, which will be denoted upper line, has a 40-km long centrally located cable section. The lower line $L_{2}$ includes a $20-\mathrm{km}$ long cable section inserted between $x=70 \mathrm{~km}$ and $x=90 \mathrm{~km}$. The two parallel-connected lines $L_{1}$ and $L_{2}$ are supplied by a $1 \mathrm{~V}$ voltage source of zero internal impedance. The load impedance is assumed infinity. The computation results are shown in Fig.4 for the current and voltage magnitudes.

Due to the charging current of the cable sections, both lines exhibit a receiving end load voltage of 1.07 per unit or volt. Their sending end currents (i.e. at $x=0$ ) are about 2.7 and $2.2 \mathrm{~mA}$ per $1 \mathrm{~V}$ of the supply voltage, respectively.

The $50 \%$ shorter cable section results in the smaller current of line $L_{2}$.

The two lines have equal receiving end currents of approximately $0.35 \mathrm{~mA}$. They have opposite phases and add up to zero. Considering the upper line, the voltage assumes a maximum value of about 1.075 per unit at $x=120 \mathrm{~km}$. As expected, the current has a zero crossing close to that co-ordinate. The greater cable capacitance per unit length, as compared with the overhead lines, is manifested in the current plots by the steeper change along the cable sections.

Fig.5 illustrates the voltage and current distributions at the frequency $f=1250 \mathrm{~Hz}$ (i.e. the $25^{\text {th }}$ harmonic). Again here, the zero current crossings occur approximately at the same locations of the voltage maxima, and vice verse. The current magnitudes along the two lines vary between zero and around $5.2 \mathrm{~mA}$. This is approximately double that of the $50-\mathrm{Hz}$ case. It is observed that the magnitude of the (anti-phased) currents at the receiving end is about $1.6 \mathrm{~mA}$. There are voltage maxima of about 1.05 per unit (at the location $x=10 \mathrm{~km}$ ) and 1.08 per unit (at $x=15 \mathrm{~km}$ ) on $L_{1}, L_{2}$, respectively.
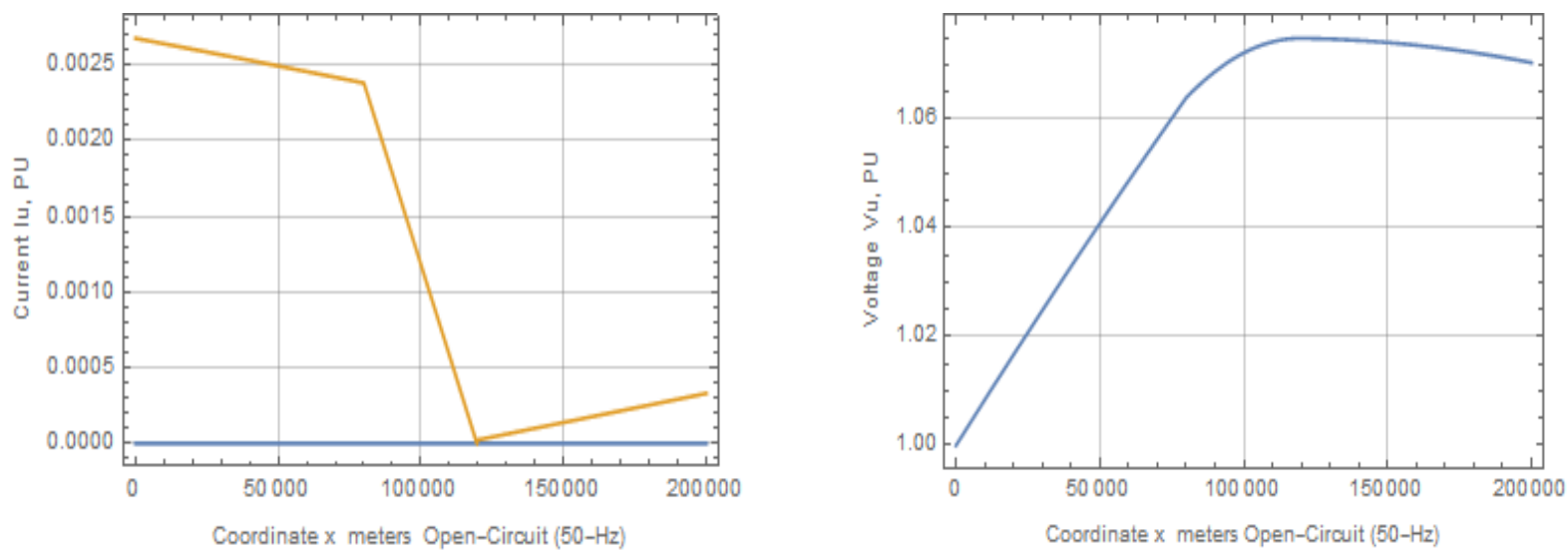

(a) along the upper line $L_{1}$ 

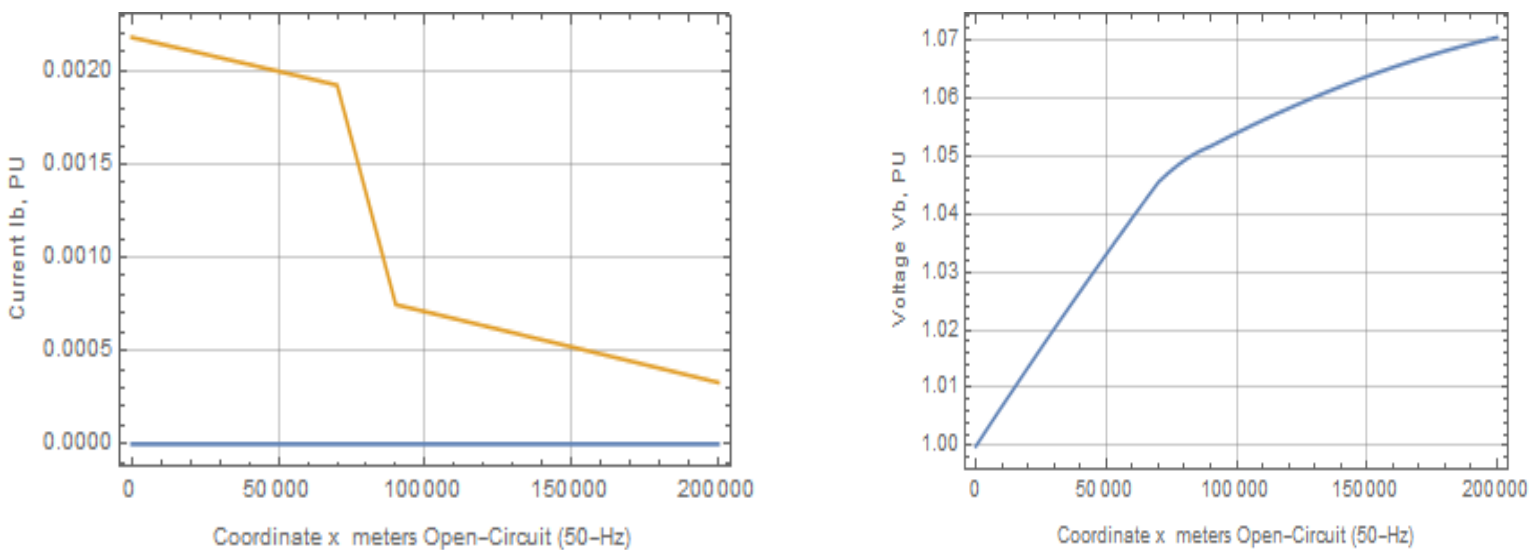

(b) along the bottom line $L_{2}$

Figure 4. The voltage and current distributions along the unloaded network of Fig 1, $(50-\mathrm{Hz})$.
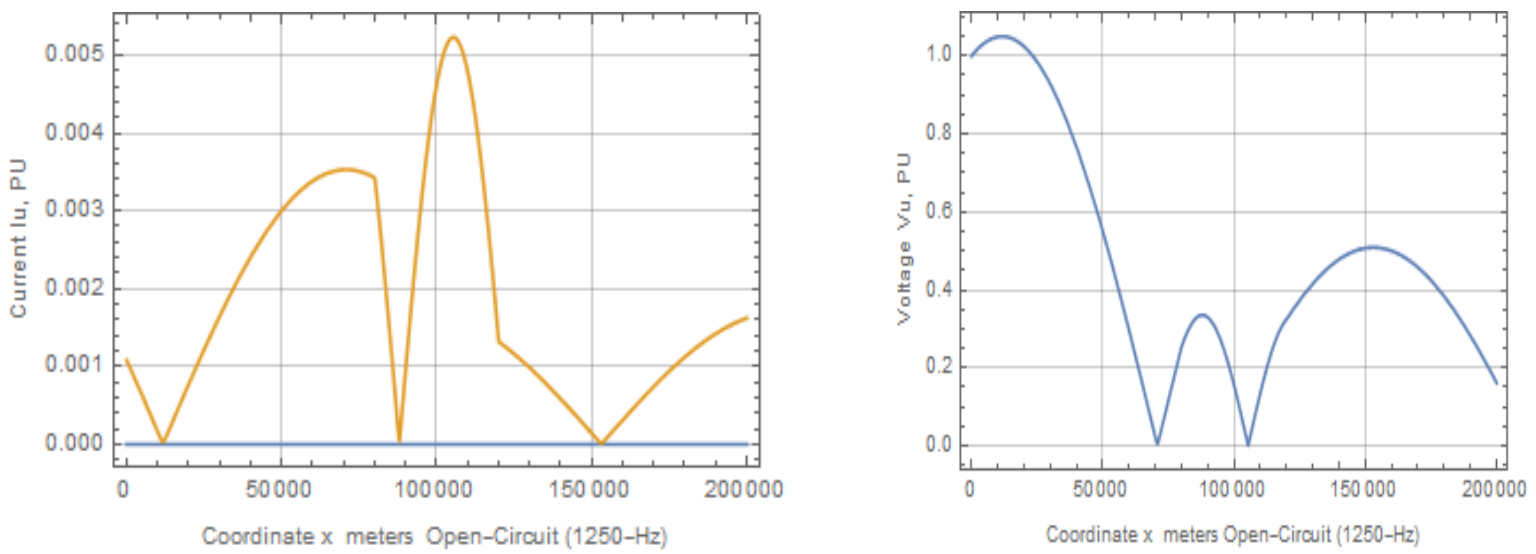

(a) along the upper line $L_{1}$
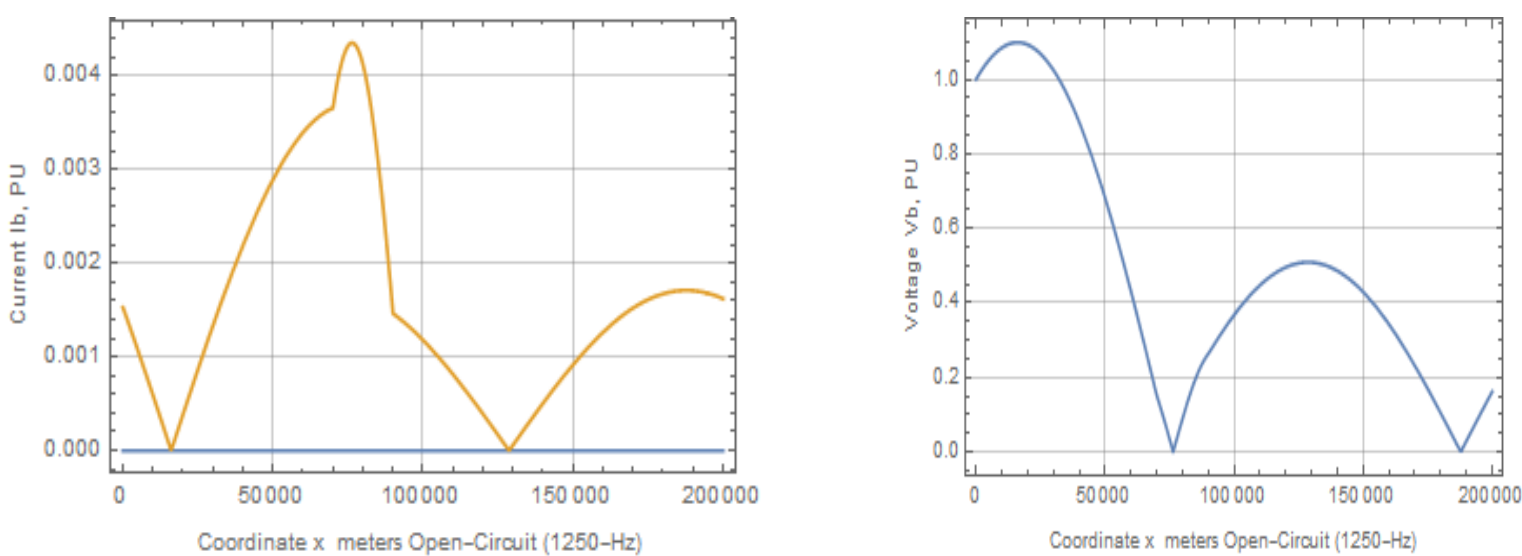

(b) along the bottom line $L_{2}$

Figure 5. The voltage and current distributions along the unloaded network of Fig 1. (1250-Hz).

The current and voltage distributions along the two lines $L_{1}, L_{2}$ of the unloaded mixed network at the frequency $5-\mathrm{kHz}$ are depicted in Fig.6. In general, there is a voltage reduction and a current increase along the inserted cable sections. The voltage at the load bus is relatively small $(0.0278-\mathrm{j} 0.00282$ per 
unit). The two receiving end currents, $i u$ and $i b$ are equal in magnitude (about $1.245 \mathrm{~mA}$ ) but opposite in phase.
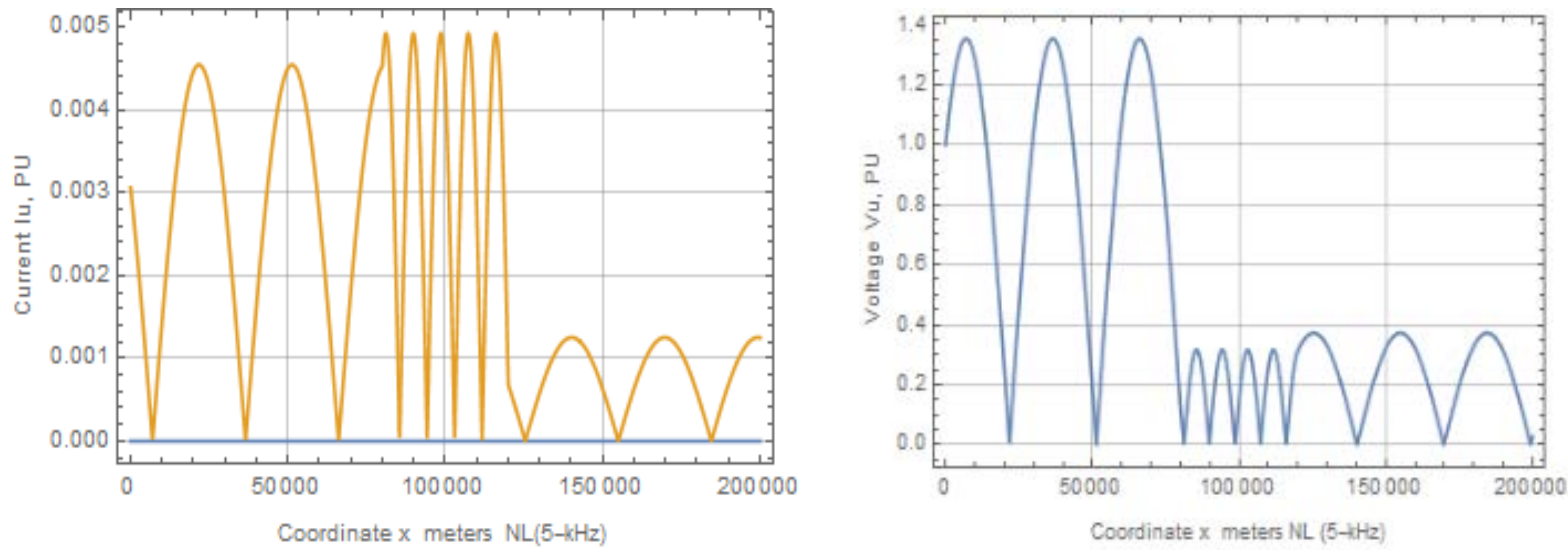

(a) distributions along the upper line $L_{1}$


(b) distributions along the bottom line $L_{2}$

Figure 6. The current and voltage distributions along the two lines $L_{1}$ (top) and $L_{2}$ (bottom) at 5-kHz at no-load.

Due to the relatively small losses, the voltage maxima and current minima occur almost at the same locations along both lines. The highest voltage and current values are approximately 1.35 per unit and $4.75 \mathrm{~mA}$. Both occur on the upper line $L_{1}$.

The wavelength along the overhead line sections is equal to the speed of light divided by the frequency, i.e. $60 \mathrm{~km}$. This can be also recognized in Fig.6.

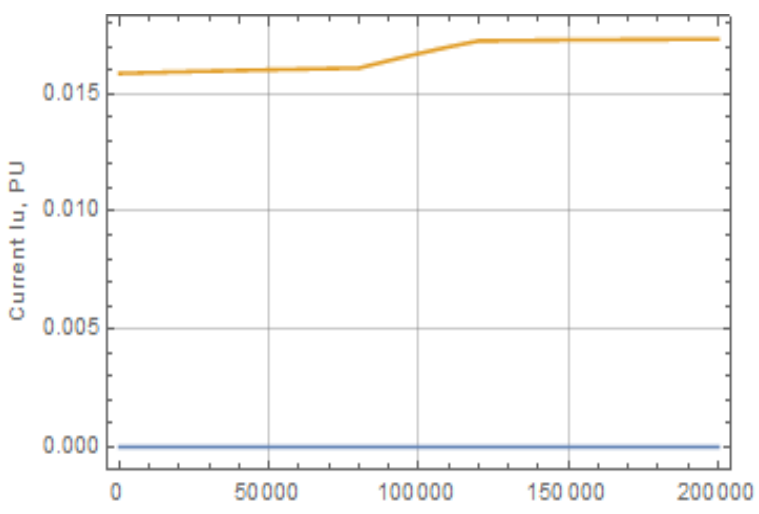

(a) Coordinate x meters Short-Circuit $(50-\mathrm{Hz})$

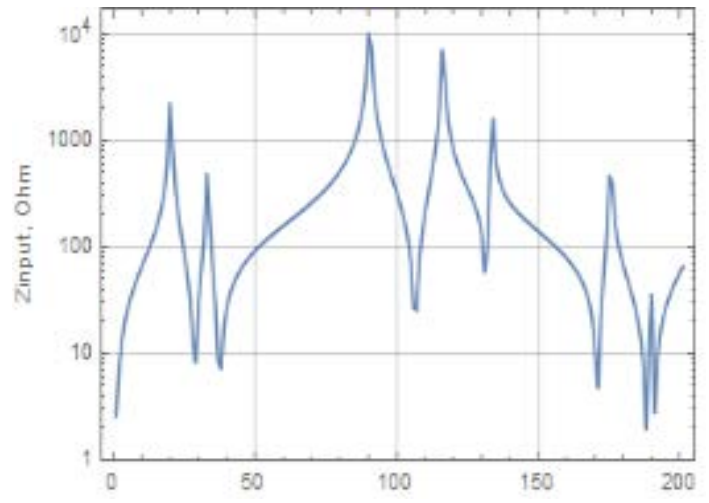

(b) Frequency. (Divide by 100)kHz Short-Circuit 


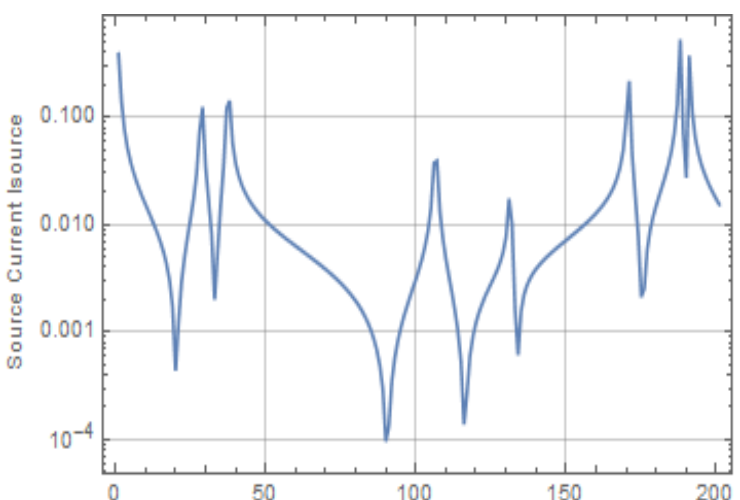

(c) Frequency. (Divide by 100) kHz Short-Circuit

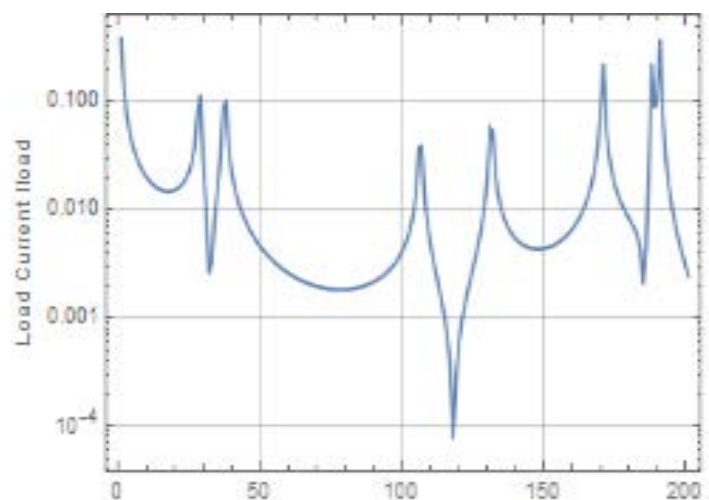

(d) Frequency. (Divide by 100) kHz Short-Circuit

Figure 7. The frequency characteristics of the voltage, current and input impedance for the upper line $L_{1}$ in the mixed network shown in Fig. 1 under short-circuit conditions.

The plots of Fig.7 describe the frequency characteristics of the considered mixed network shown in Fig. 1 with a short-circuited receiving end. The chart (a) illustrates the current distribution along the line at $50-\mathrm{Hz}$. It increases from approximately $16 \mathrm{~mA}$ near the source terminal to about $17.5 \mathrm{~mA}$ near the receiving end. Most of the increase occurs along the cable section, i.e. between $x=80$ and $120 \mathrm{~km}$. The plot (b) illustrates the magnitude of the network's input impedance over the frequency range from zero to $2 \mathrm{kHz}$. Under DC conditions, the input impedance is equal to the resistance of the parallel-connected lines $L_{1}, L_{2}$. The first parallel resonance occurs at about $200 \mathrm{~Hz}$ resulting in the relatively high input impedance of $1.4 \mathrm{k} \Omega$. This corresponds to the small source current of $0.7 \mathrm{~mA}$, as seen in the plot (c). The large value of the source current at zero frequency (above $200 \mathrm{~mA}$ ) is only limited by the network's resistances and conductances. The plot indicates also the possibility of a network's parallel resonance close to the seventh harmonic frequency $350 \mathrm{~Hz}$. The series resonance close to $1700 \mathrm{~Hz}$ can be also clearly recognized. The frequency response of the load current under short-circuit, plot (d), depicts a very clear parallel resonance near $1200 \mathrm{~Hz}$ resulting in the small current of $0.08 \mathrm{~mA}$.
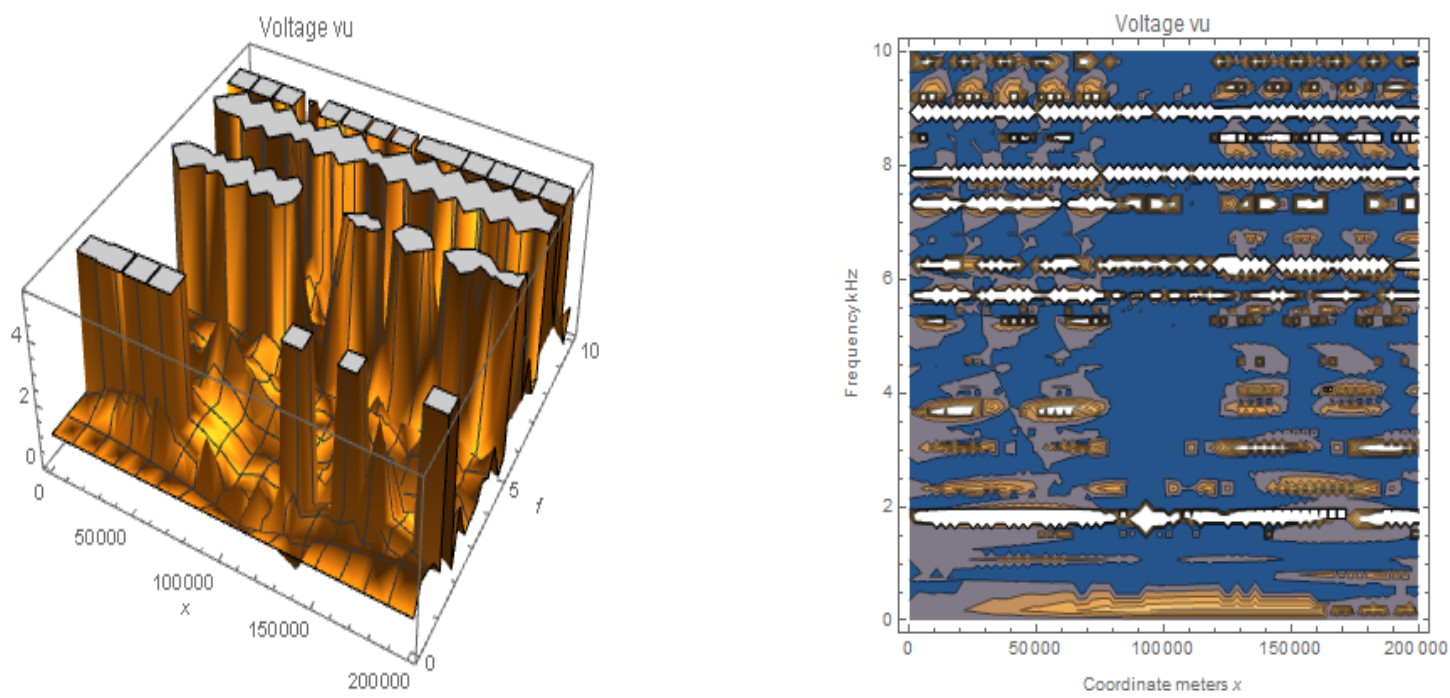

Figure 8. Three-dimensional and contour plots of the voltage $v u$ along the line $L_{1}$ as a function of both the coordinate $x$ (in meters) and the frequency $f$ (in $\mathrm{kHz}$ ) under no-load conditions.

In order to demonstrate the possible application of the suggested procedure, the developed Mathematica program was used to generate the 3-D and contour plots depicted in Fig.8. They illustrate 
the distribution of the voltage magnitude along the 200-km long upper line $L_{1}$ over the frequency range from zero to $10 \mathrm{kHz}$. It can be seen from the 3 -D plot that under DC conditions $(f=0)$ the voltage is equal to $v u=1$ per unit over the entire line, as expected. Both plots indicate that, at certain frequencies, the voltage can assume relatively high values at certain points along the line. At the frequencies $f=1.8$, 5.6 and $8.9 \mathrm{kHz}$, for instance, most of the line will be subject to a voltage magnification. More details are shown in Fig.9 for the current and voltage distributions along both lines at $f=1.8 \mathrm{kHz}$. The current values can exceed $40 \mathrm{~mA}$ per $1 \mathrm{~V}$ of the supply voltage. On both lines, excessive voltage values of more than 9.5 times the source voltage will appear at points approximately $40 \mathrm{~km}$ far from the source bus.

The interesting special case of the network's surge impedance loading was also investigated. Since each of the two overhead lines has a surge impedance of $Z_{o, \text { line }}=297.515 \Omega$, the condition of impedance matching will be approximately satisfied if the load impedance is pure resistive and equal to half of this value (neglecting losses). The voltage and current distributions are given in Fig. 10 for the fundamental frequency $f=50 \mathrm{~Hz}$. The voltage distributions show almost flat profiles with slight deviations from the source voltage 1 per unit. This is not the case for the two plots describing the magnitude of the currents along the lines. The current magnitude along $L_{1}$ drops from $4.2 \mathrm{~mA}$ at the sending end to $3.6 \mathrm{~mA}$ at the load terminal: The corresponding values for the line $L_{2}$ are $3.8 \mathrm{~mA}$ at $x=0$ and $3.4 \mathrm{~mA}$ at $x=200 \mathrm{~km}$, respectively. As the plots indicate, the changes in the current magnitude occur mainly along the inserted cable sections.

It should be noted that the plots represent the magnitudes and not the instantaneous values. The plots under DC conditions (not given here due to space limitations) indicate almost flat profiles for both currents and voltages. The voltage magnitude is almost constant throughout the network and is very close to unity (the deviations are due to the loss dissipation). The current magnitudes are uniform (approximately $3.35 \mathrm{~mA}$ ) along each of the two 200-km lines $L_{1}, L_{2}$. This value agrees with the DC analysis taking into account that each of the two parallel connected lines has a resistance of $5.2 \Omega$ and that the matched load impedance is $297.515 \Omega$.
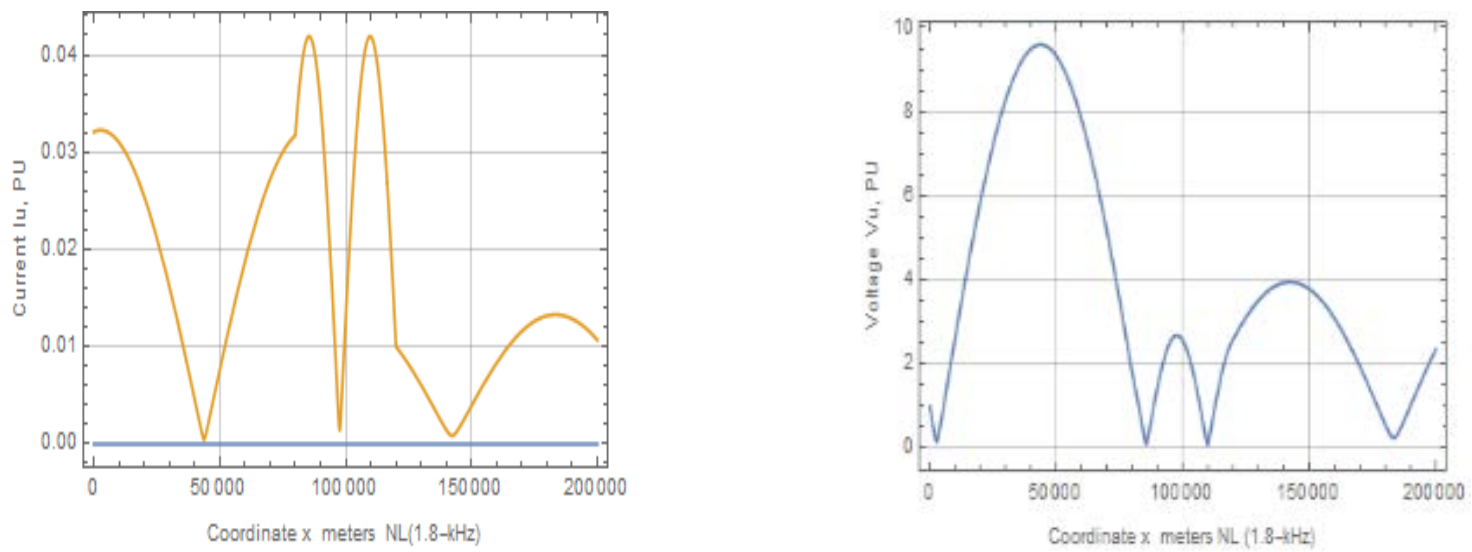

(a) distributions along the upper line $L_{1}$ 

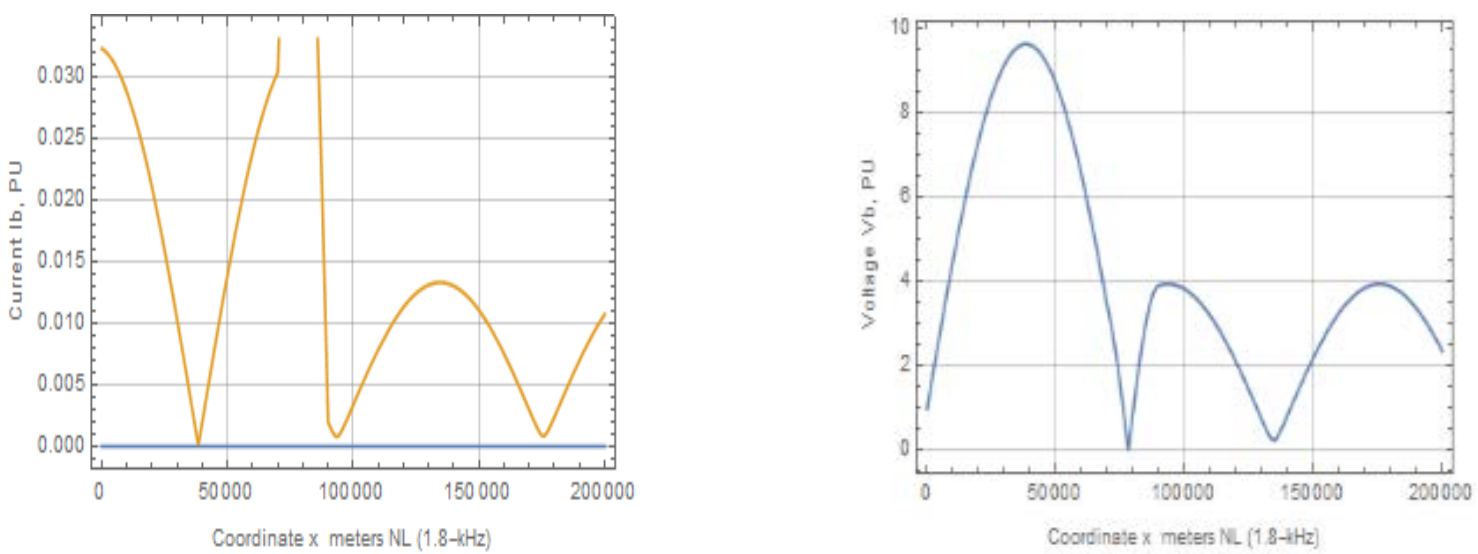

(b) distributions along the bottom line $L_{2}$

Figure 9. The current and voltage distributions along both lines at $f=1.8 \mathrm{kHz}$.
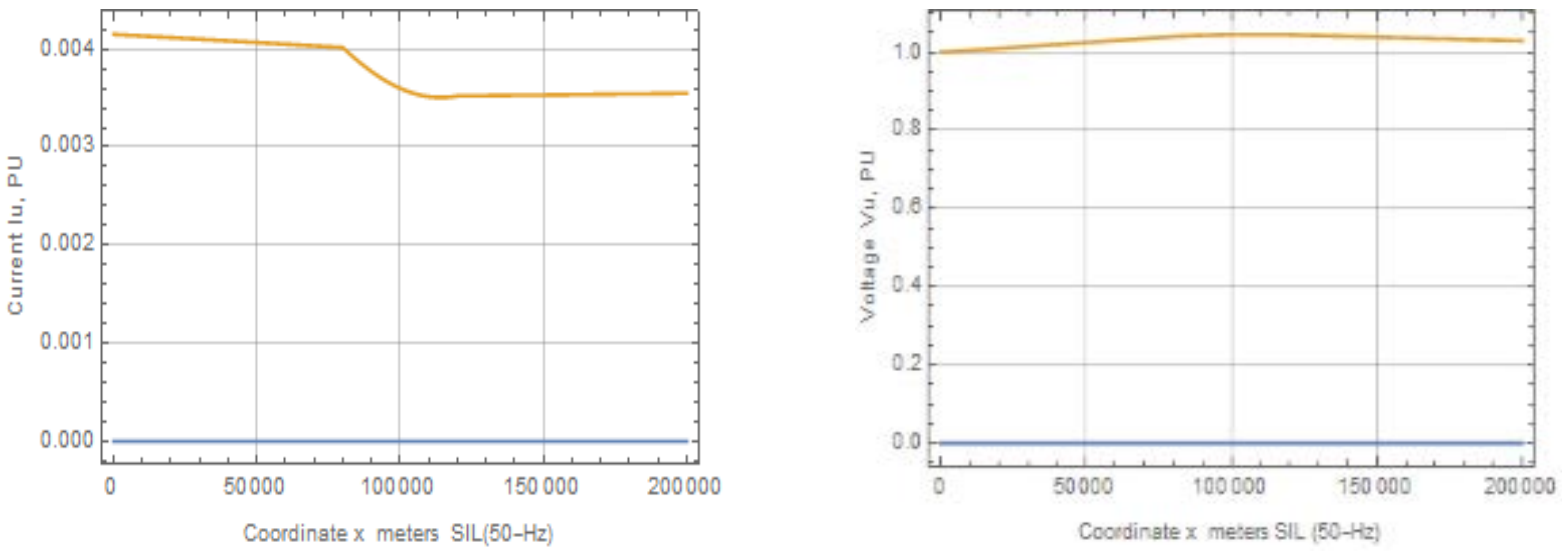

(a) distributions along the upper line $L_{1}$
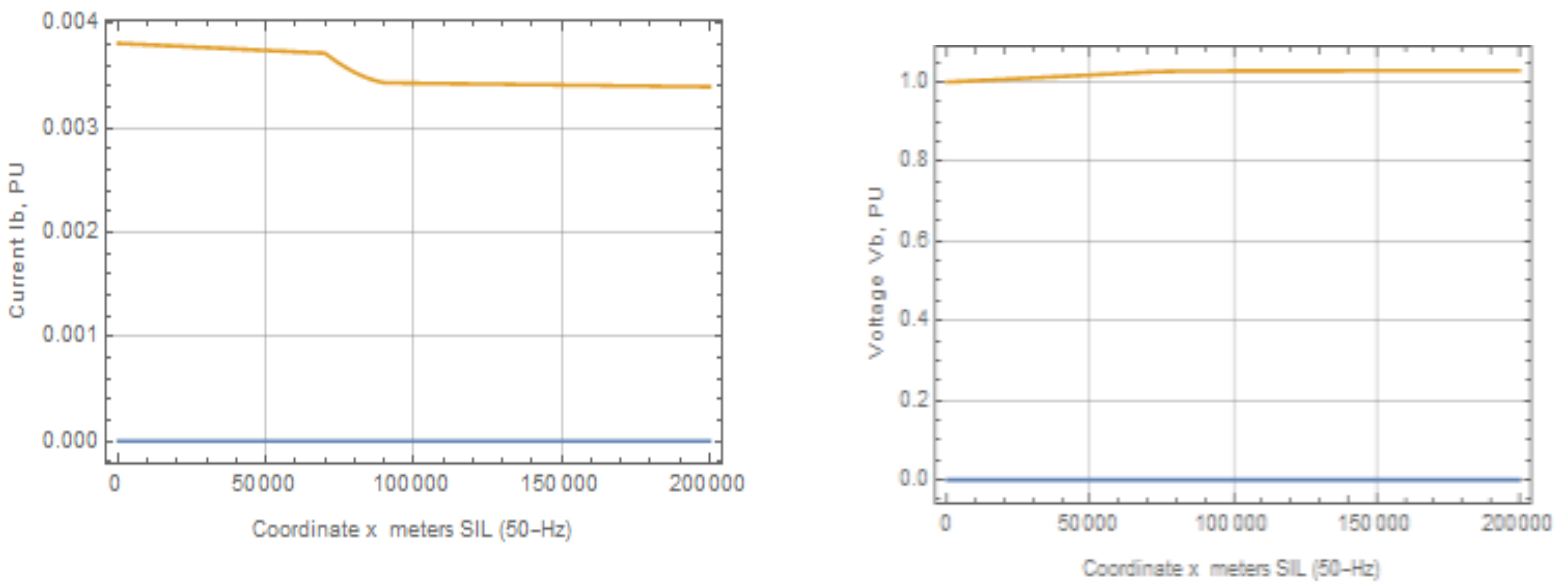

(b) distributions along the bottom line $L_{2}$

Figure 10. The current and voltage distributions along both lines at $f=50 \mathrm{~Hz}$ and a resistive load of $Z_{\text {load }}=Z_{\text {oline }} / 2$. 


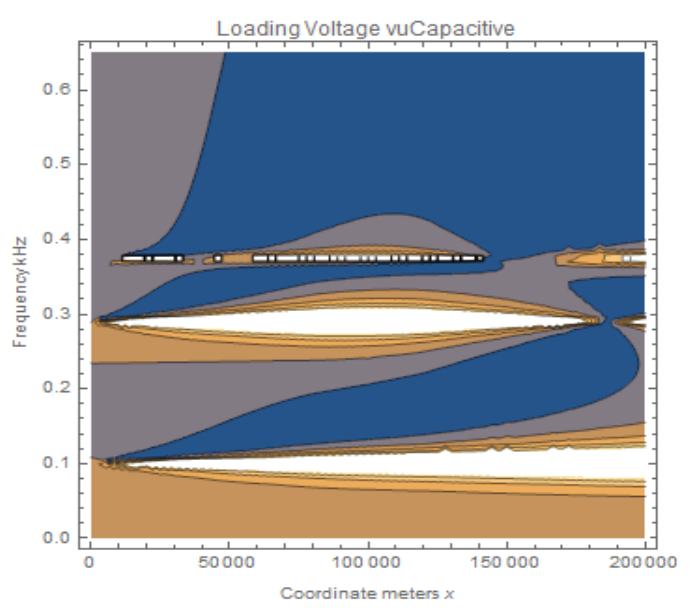

(a) Contour plot of the voltage $v u$ along $L 1$ versus frequency $f$

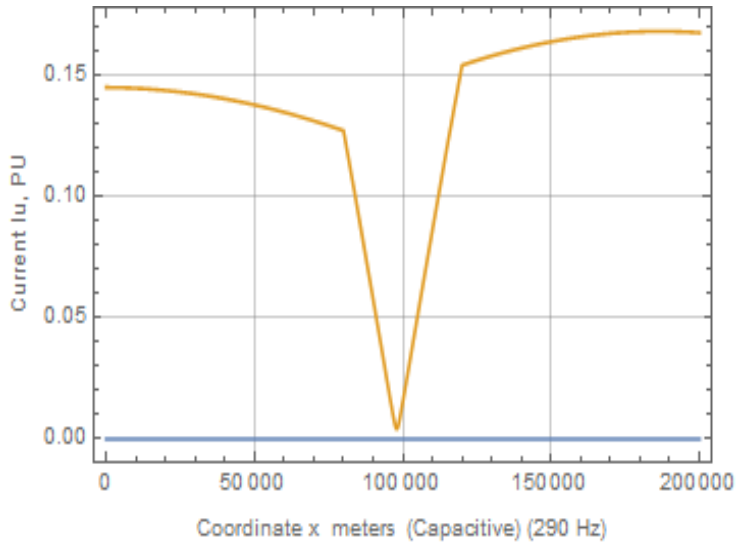

(c) Current distribution along $L 1$ at $290 \mathrm{~Hz}$

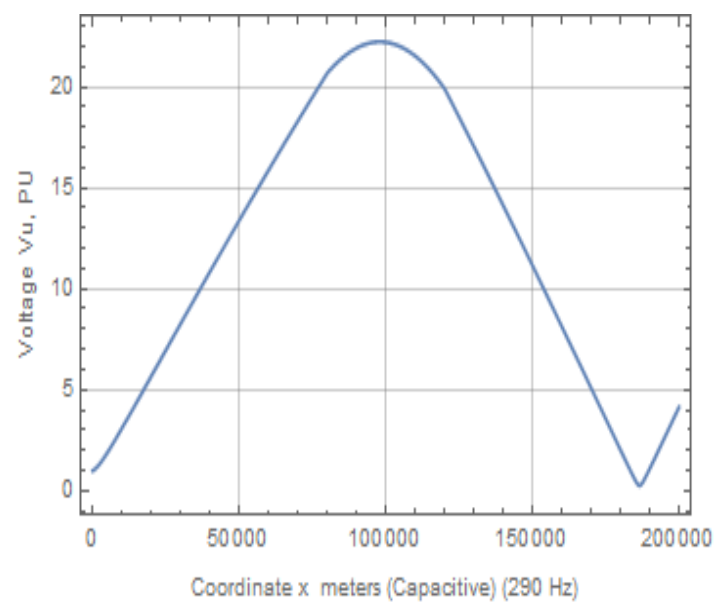

(b) Distribution of the voltage $v u$ along $L 1$ at $290 \mathrm{~Hz}$

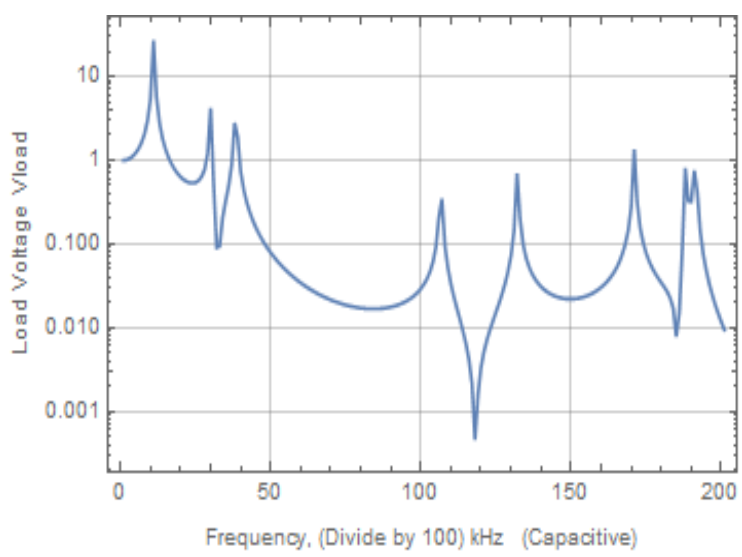

(d) the load voltage vload versus frequency

Figure 11. The network's frequency characteristics when the network is loaded by a $21.4 \mu F$ pure capacitive impedance.

Fig.11 illustrates the results pertinent to the case of loading the mixed network by a pure capacitive impedance. The load capacitance is assumed $21.4 \mu \mathrm{F}$, corresponding to a $50-\mathrm{Hz}$ reactance of $148.5 \Omega$. This is numerically equal of the combined surge impedance of the two parallel connected lines. The contour plot in the upper left plot (a) depicts the voltage $v u$ along the line $L 1$ as a function of both the co-ordinate $x$ and the frequency $f$. The brightness at any point is proportional to the voltage magnitude. The considered frequency range extends from zero to $650 \mathrm{~Hz}$ (the $13^{\text {th }}$ harmonic). It is noticed that there are certain frequencies, such as $100 \mathrm{~Hz}$, at which the entire line $L 1$ exhibits serious voltage magnification. At some other frequencies only some parts of the line are subject to voltage concentration. This is the case at $290 \mathrm{~Hz}$ depicted by the voltage and current plots (b) and (c), respectively. The voltage magnitude close to the line midpoint (also the midpoint of the cable section in this particular case study) reaches almost 22.2 times the source voltage. This will result in large shunt capacitive currents along the cable section, i.e. between $x=80$ and $120 \mathrm{~km}$. This is noticeable in plot (c), where the current at the cable junctions steeply drops from $125 \mathrm{~mA}$ to $152 \mathrm{~mA}$ in the opposite direction with a zero crossing at $x=98 \mathrm{~km}$. Plot (d) illustrates the frequency dependence of the load voltage vload. Under DC conditions, the currents throughout the mixed network are zero, and the load and source voltages will be equal. The highest voltage magnification of about 23 per unit occurs at $f=100 \mathrm{~Hz}$. At the frequency $f=1190 \mathrm{~Hz}$, an extremely low load voltage of less than 0.0005 per unit of the supply voltage will result. 


\section{Model Validation and Discussion of the Results}

The suggested approach is validated through its application to the following three case studies:

\subsection{Case A}

The Mathematica program is applied to analyze an unloaded lossless power network composed of two identical 200-km long parallel connected overhead lines without any cable sections, i.e. $w_{1}=w_{2}=0$. The source impedance is assumed zero. The $50-\mathrm{Hz}$ supply voltage is 1.0 per unit. The plot in Fig. 12-(a) depicts the voltage profile along any of the two lines as obtained from the computer program based on the suggested technique. Due to the Ferranti effect, the voltage $v(x)$ increases gradually with the distance $x$ measured from the source bus. The voltage at the load is about 1.026 per unit. This numerical value and the entire curve in Fig.12-(a) are in full agreement with the following exact analytical expression derived from transmission line theory:

$$
v(x)=\frac{\cos \beta(\text { Length }-x)}{\cos \beta \text { Length }}
$$

where $\beta$ is the line's phase constant $=\omega \sqrt{l c}$ and Length is the entire line length $=200000$ meters. The corresponding results are depicted in Fig. 12-(b). For more details, Reference [17] should be consulted.

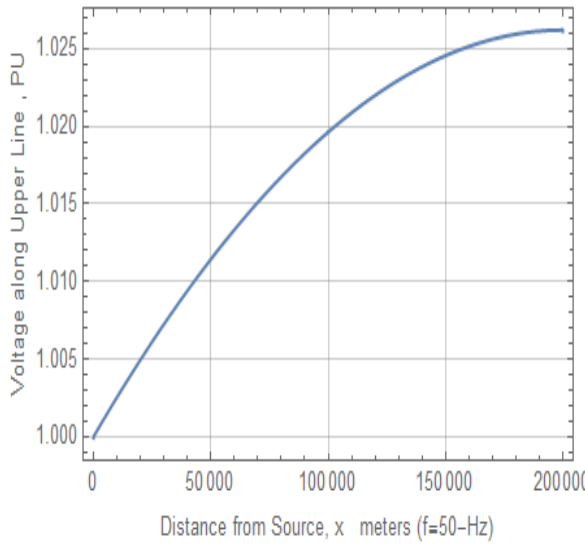

(a) Results from the suggested technique

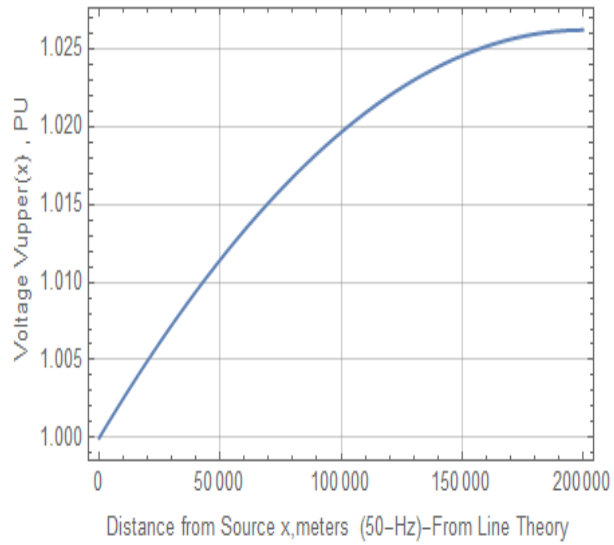

(b) Results from line theory

Figure 12. The voltage distribution along the lines $L_{1}, L_{2}$ at no-load.

\subsection{Case B}

The plot given in Fig.13 illustrates the distribution of the voltage magnitude along the upper line $L_{1}$ at the frequency $f=1.8-\mathrm{kHz}$ under no-load conditions. It is obtained through the application of the classical two-port equations and the well-known transmission line $A B C D$ constants of the six two-port networks depicted in Fig.2. This plot corresponds to the one given earlier in Fig. 9-(a) which was the result of applying the suggested technique. The agreement between the two curves can be recognized.

It should be noted that the efficiency of the classical solution depends primarily on the accuracy of solving 16 simultaneous complex algebraic equations. On the other hand, the accuracy of the suggested technique depends on the method used in the Mathematica program for solving only four simultaneous differential equations governing the lines' voltage and currents. 




Figure 13. Voltage distribution along the upper line $L_{1}$ at $1.8-\mathrm{kHz}$ under no-load conditions resulting from applying the method of two-port networks.

\subsection{Case $\mathrm{C}$}

The mixed network is analyzed under DC conditions. The lengths of the two lines $\mathrm{L}_{1}$ and $\mathrm{L}_{2}$ are assumed 100 and $150 \mathrm{~km}$ long, respectively. Their combined equivalent resistance is therefore $12 \Omega$. The network's load is assumed $88 \Omega$, and the source internal resistance is substituted as zero. The total equivalent resistance seen by the source is $12+88=100 \Omega$. The Mathematica program results for the different currents resulting from a source voltage of 1.0 per unit are as follows:

The source current $\mathrm{L}_{1}=0.010 \mathrm{~A}$, The load current $\mathrm{L}_{2}=0.010 \mathrm{~A}$

All currents along $\mathrm{L}_{1}=0.006 \mathrm{~A}$, All currents along $\mathrm{L}_{2}=0.004 \mathrm{~A}$

These values are exactly equal to those resulting from simple DC analysis. The same observation is noticed regarding the voltage distribution.

It should be noted, however, that the suggested direct procedure is superior in terms of the required computational resources. In summary, it is a much faster alternative that needs less computer memory to program.

\section{Conclusions}

The concept of the partial undergrounding of some overhead transmission lines through the insertion of suitable cable sections is addressed. The economical and technical impacts on the resulting mixed power networks are discussed and a model for analyzing their frequency characteristics is presented. For comparison, the classical method of the analysis by applying the theory of two-port networks and the generalized circuit constants of transmission lines is also described. The paper suggests an alternative direct solution technique based on representing all sections of each partially undergrounded line by a single equivalent non-uniform line. Its location dependent circuit parameters can be described by analytical expressions utilizing suitable collections of step functions. This approach yields the voltages and currents at the line-cable junctions, as well as the internal distributions within the line and cable sections. Each equivalent non-uniform line is described by two simultaneous differential equations for its voltage and current. These equations will be augmented by the loop and node equations expressing the interconnection of the lines with the sources and loads. A direct solution could then be obtained comprising several parametric functions in terms of the co-ordinates, the line and cable parameters, the locations and lengths of each of the inserted cable sections, the frequency as well as the network's loading condition. The suggested technique is applied to a number of case studies in order to explore the effect of the above factors on the voltage and current distributions. The paper discusses also the frequency characteristics of the input impedance. The results indicate that, depending on the frequency and the loading condition of the mixed network, excessive currents and voltages can appear at certain locations along the lines and their cable sections. It is also observed that the locations of the voltage maxima coincide with those of the current zeros, and vice verse. 
It is believed that the presented approach can assist the practicing engineer in quickly identifying possible issues resulting from the partial undergrounding such as the expected values and locations of voltage and current concentrations at certain frequencies.

\section{References}

1. L. Colla, F. M. Gatta, F. Iliceto, S. Lauria: "Design and operation of EHV transmission lines including long insulated cable and overhead sections", Presented at the $7^{\text {th }}$ IPEC Power Engineering Conference, 2005.

2. P. H. Larsen:"A Method to Estimate the Costs and Benefits of Undergrounding Electricity Transmission and Distribution lines", Preprint of a manuscript accepted at Energy Economics. October 2016.

3. ENTSO-E and Europacable: Joint paper:"Feasibility and technical aspects of partial undergrounding of extra high voltage power transmission lines", Brussels, December 2010.

4. The National Grid House:"Undergrounding high voltage electricity transmission lines", Warwick Technology Park Gallows Hill WarwickCV34 6DA www.nationalgrid.com, Issue 4: January 2015.

5. R. Benato, A.Paolucci:"EHV AC Undergrounding Electrical Power Performance and Planning", Book, SpringerVerlag London Limited 2010.

6. F.M. Gatta, A. Geri, S. Lauria, M. Maccioni: "Power frequency secondary arc current in uncompensated EHV AC mixed cable-overhead lines" Journal of Electric Power Systems Research, 111, pp. 14-21, 2014.

7. H.Khalilnezhad, M.Popov, J. A.Bos, Kees P.J. Jansen:" Influence of partial undergrounding on the transient stability of EHV power transmission systems" Journal of Electric Power Systems Research, 131, pp. 26-138, 2016.

8. L.Wu:" Impact of EHV/HV underground power cables on resonant grid behavior", PhD. Thesis. Technische Universiteit Eindhoven, Available on line at http://alexandria.tue.nl/extra2/781433.pdf, 2014.

9. O.Galland, D.Leu, V.Berner, P. Favre-Perrod: "Resonance Analysis of a Transmission Power System and Possible Consequences of its Undergrounding", Period. Polytech. Elec. Eng. Comp. Sci., Vol. 59, No. 3 (2015), pp. 88-93.

10. F. M. F. Da Silva: "Analysis and simulation of electromagnetic transients in HVAC cable transmission grids" PhD. Thesis, Aalborg University, Denmark, 2011.

11. M. Kizilcay:"Switching Overvoltages in a 400-kV Cable System", Presented at The 16th PSCC, Glasgow, Scotland, July 14-18, 2008

12. M. M. Saied: "Effect of Cable Sections on the Electromagnetic Transients in Power Networks" Journal Electric Machines 8 Power Systems, Volume 15, 1988, Issue 1, Pages 17-35.

13. M. M. Saied: "Modifying the Transient Overvoltages in Mixed Power Networks by Inserting Cable Sections", Electrical Power Quality and Utilization Journal, (EPQU), Vol. 18, Issue 1, pp. 7-14, 2015. Also available at: https://www.researchgate.net/publication/281457743_Modifying_the_Transient_Overvoltages_in_Mixed_Pow er_Networks_by_Inserting_Cable_Sections.

14. J.Grainger, W.D.Stevenson.Jr.:"Power System Analysis", Book, McGraw-Hill International Edition, 1994.

15. Wolfram Research: Documentation on the software Mathematica 10, available at: http://www.wolfram.com/mathematica/?source=nav, 2014.

16. Wolfram Mathematica Tutorial Collection. Advanced Numerical Differential Equation Solving in Mathematica, Book, Wolfram Research Inc., 2008, available at:

http://www.wolfram.com/learningcenter/tutorialcollection/AdvancedNumericalDifferentialEquationSolvingInMat hematica/

17. T.J.E. Miller:" Reactive Power Control in Electric Systems", Book, John Wiley \& Sons, Chapter 2, 1982. 